\title{
Molecular packing in cadmium and zinc arachidate LB multilayers
}

\author{
N. Prasanth Kumar a, S. Major ${ }^{\text {a,* }}{ }^{\text {, Satish Vitta }}{ }^{\text {b }}$, S.S. Talwar ${ }^{\text {c }}$, P. Dubcek ${ }^{\mathrm{d}}$, \\ H. Amenitsch ${ }^{\mathrm{d}}$, S. Bernstorff d, V. Ganesan ${ }^{\mathrm{e}}$, Ajay Gupta ${ }^{\mathrm{e}}$, \\ B.A. Dasannacharya ${ }^{\mathrm{e}}$ \\ a Department of Physics, Indian Institute of Technology Bombay, Mumbai 400 076, India \\ ${ }^{\mathrm{b}}$ Department of Metallurgical Engineering and Materials Science, Indian Institute of Technology Bombay, Mumbai 400 076, India \\ c Department of Chemistry, Indian Institute of Technology Bombay, Mumbai 400 076, India \\ d Sincrotrone Trieste, 34012 Basovizza (TS), Trieste, Italy \\ e Inter University Consortium for Department of Atomic Energy Facilities, University Campus, Indore 452 017, India
}

\begin{abstract}
A combination of grazing incidence X-ray reflection/diffraction (GIXR/GIXD) and atomic force microscopy have been used to study the 3D structure in cadmium arachidate (CdA) and single phase zinc arachidate (ZnA) LB multilayers. The CdA multilayers have an ideal close packed herringbone structure. The molecules have a non-centred in-plane arrangement with specific orientational relation between the central and the corner molecules. In contrast, the $\mathrm{ZnA}$ multilayers have a hexagonal layer plane packing with tilted molecules. The molecules are loosely packed with rotational freedom about chain axis as observed in 'rotator' phases.
\end{abstract}

Keywords: Langmuir-Blodgett multilayers; Cadmium arachidate; Zinc arachidate; AFM; Molecular packing

\section{Introduction}

Long-chain fatty acids and their divalent arachidate/stearate salts have been the most extensively studied LB systems, among which, cadmium- and lead-substituted monolayers and multilayers have attracted maximum attention [1-
3]. The intralayer structure and molecular packing in these systems have been studied by a variety of techniques, such as, electron diffraction [4,5], grazing angle X-ray scattering [6-9] and atomic force microscopy (AFM) [10,11]. Grazing incidence X-ray diffraction (GIXD) studies of Cd and $\mathrm{Pb}$ arachidate/stearate multilayers have shown that the intralayer molecular packing corresponds to a centred rectangular or distorted hexagonal two-dimensional (2D) structure with molecules nearly perpendicular to the layer plane [7-9]. 
These observations have been supported by AFM studies [10,11], which have shown that for both $\mathrm{Cd}$ and $\mathrm{Pb}$ arachidate/stearate multilayers, the $2 \mathrm{D}$ in-plane structure consists of a two molecule rectangular unit cell with herringbone packing of all-trans alkane chains. Both GIXD and AFM studies have independently shown that the rectangular layer cell parameters are close to $0.496 \mathrm{~nm}$ by $0.742 \mathrm{~nm}$, which are the layer cell dimensions proposed by Kitaigorodskii [12] for $\mathrm{R}[0,0]$, the ideal close packed untilted molecular packing in long chain organic compounds. Extension of the AFM studies [10] to a variety of fatty acid salts such as $\mathrm{Mg}, \mathrm{Mn}, \mathrm{Ca}, \mathrm{Ba}$ and $\mathrm{Zn}$ arachidates/ stearates has been used to further conclude that as the electronegativity of the metal ion decreases, the bonding between the metal ion and alkyl chain ending in the carboxylate ion tends to become ionic. The non specific nature of ionic bonding has been associated with a large headgroup area required per molecule which in turn induces the alkyl chains to tilt and keep the van der Waals chain-chain interaction intact in the plane normal to the chains. The molecular packing in the divalent metal fatty acid salt multilayers has thus been shown to depend systematically on the electronegativity of the metal ion and in most cases, corresponds to the close packed structures proposed by Kitaigorodskii [12] for long-chain organic compounds. However, it has been reported [13] that zinc arachidate ( $\mathrm{ZnA})$ multilayers do not follow this trend and the molecules pack in a distorted hexagonal layer cell with chains tilted at $\sim 30^{\circ}$ towards the nearest neighbour. In a more recent study [14], it has been further shown that the layered structure in $\mathrm{ZnA}$ multilayers depends strongly on subphase $\mathrm{pH}$ at which the monolayers are transferred and exhibits polymorphic phases with different types of layered structures. It was also found that only at a subphase $\mathrm{pH}$ of $\sim 6.5$ the $\mathrm{ZnA}$ multilayer exhibits a nearly single phase layered structure. Since $\mathrm{Cd}$ and $\mathrm{Zn}$ have similar electronegativity values, 1.7 and 1.6, respectively, but show very different packing behaviour as observed by AFM, the arachidates of these metals were chosen in the present work for a complete
3D structural study. The structural studies in LB multilayers have so far been done using either $\mathrm{X}$-ray scattering or AFM. AFM is a surface sensitive technique while $\mathrm{X}$-ray scattering provides volume structural information. A combination of these techniques, namely grazing incidence X-ray reflectivity (GIXR), grazing incidence X-ray diffraction (GIXD) and AFM has been used in this work to investigate the $3 \mathrm{D}$ structure of $\mathrm{CdA}$ and single phase $\mathrm{ZnA}$ multilayers.

\section{Experimental}

Cadmium arachidate and zinc arachidate multilayers were made by the conventional LB deposition technique using a KSV 3000 LB instrument in a clean room. Arachidic acid (Aldrich, 99\%) with HPLC grade chloroform as solvent (1 $\mathrm{mg} \mathrm{ml^{-1 }}$ ) was used to spread the monolayer on Millipore ultrafiltered water (resistivity of 18.2 $\mathrm{M} \Omega \mathrm{cm})$ subphase. $\mathrm{CdCl}_{2}\left(4 \times 10^{-4} \mathrm{M}\right)$ and $\mathrm{ZnCl}_{2}\left(10^{-5} \mathrm{M}\right)$, respectively, were added to the subphase and the $\mathrm{pH}$ was adjusted to $\sim 6.5$ by adding $\mathrm{NaHCO}_{3}$. The pressure-area isotherms in both cases were of condensed nature without a liquid condensed region, as shown in an earlier study [14]. The limiting mean molecular areas for $\mathrm{CdA}$ and $\mathrm{ZnA}$ monolayers were found to be 0.20 and $0.202 \mathrm{~nm}^{2}$, respectively. The monolayers were transferred onto quartz substrates which were cleaned to remove both organic and inorganic impurities using standard procedures. Multilayers were transferred at a constant surface pressure of $30 \mathrm{mN} \mathrm{m}^{-1}$ and a vertical dipping speed of 3 $\mathrm{mm} \mathrm{min}^{-1}$ at room temperature $\left(20^{\circ} \mathrm{C}\right)$. A total of 25 monolayers were transferred for all the studies with near unity transfer ratios. FTIR spectra of $\mathrm{CdA}$ and $\mathrm{ZnA}$ multilayers showed that in both cases, the films consisted only of arachidate salts with no arachidic acid component.

The X-ray reflectivity measurements were carried out on a Siemens D5000 diffractometer with $\mathrm{CuK}_{\alpha}$ radiation. A razor blade creates a slit of $\sim 0.02 \mathrm{~mm}$ at the sample and results in $0.02^{\circ}$ divergence of the incident beam. The exit beam 
path consists of a soller slit, LiF (100) monochromator and a NaI scintillation detector. The GIXD studies were performed using synchrotron radiation of wavelength $0.155 \mathrm{~nm}$ at the Italian synchrotron source ELETTRA (SAXS beamline). An asymmetrically cut $\mathrm{Si}(111)$ double crystal both selects and monochromatizes the $0.155 \mathrm{~nm}$ radiation. A guard slit of size $2.0 \mathrm{~mm} \times 0.05 \mathrm{~mm}$ $(\mathrm{HxV})$ defines the illuminated area on the sample surface. A 1D gas filled detector which has an angular resolution of $0.05^{\circ}$ was used to detect the diffracted beam whose size was defined by a 1.6 $\mathrm{mm} \times 0.6 \mathrm{~mm}$ exit slit. The angle of incidence was kept close to the critical angle for total reflection, $\sim 0.3^{\circ}$ and the diffraction spectrum was studied in the range $10-60^{\circ}$. AFM measurements were performed with a Nanoscope E (Digital Instruments) in air at room temperature. A silicon nitride tip with a spring constant of $\sim 0.58 \mathrm{~N} \mathrm{~m}^{-1}$ was used. The AFM measurements were performed in 'contact' mode to obtain molecular resolution images. In order to avoid thermal drifts, scans were performed at a rate of $10-30 \mathrm{~Hz}$ and the mechanical drifts were eliminated by performing the experiments in a vibration-isolated arrangement.

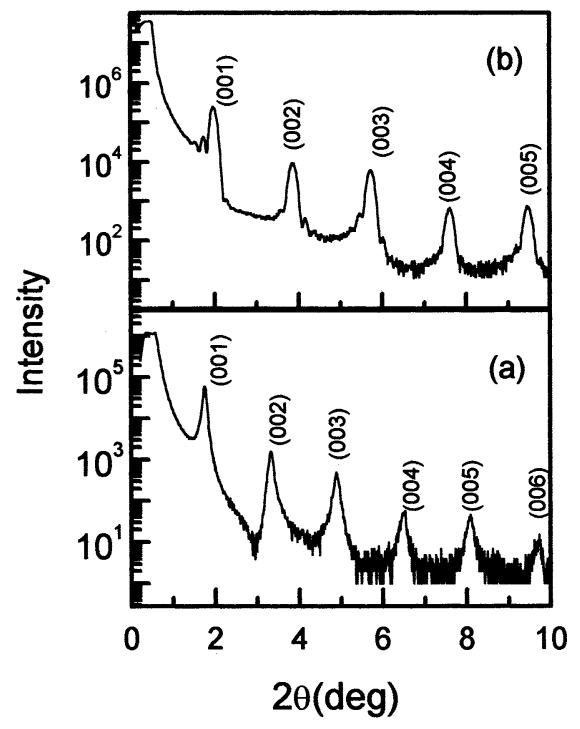

Fig. 1. The grazing incidence X-ray reflectivity from the: (a) CdA; and (b) ZnA multilayers shows clear Bragg peaks, indicative of a well-formed layered structure.

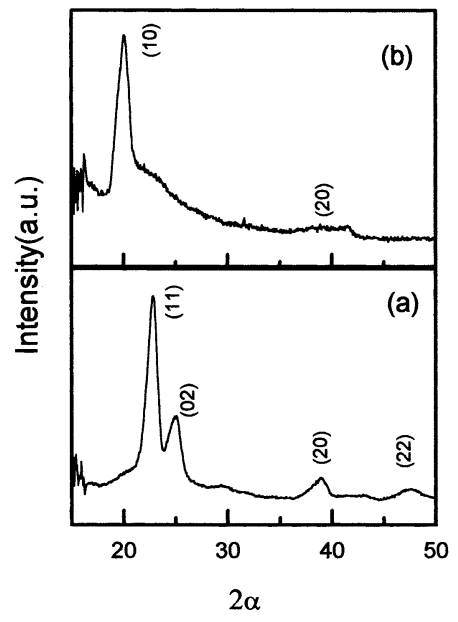

Fig. 2. The grazing incidence $\mathrm{X}$-ray diffraction patterns from the: (a) $\mathrm{CdA}$; and (b) $\mathrm{ZnA}$ multilayers in the range of $15-50^{\circ}$ shows peaks indicating the presence of crystallinity in the layer plane.

\section{Results and discussion}

Fig. 1 shows the X-ray reflectivity scans of the $\mathrm{CdA}$ and $\mathrm{ZnA}$ multilayers transferred onto quartz substrates. The presence of up to 5 orders of $(00 l)$ Bragg peaks in both cases indicates the formation of well-defined layered structures along the growth direction. The bilayer period can be determined from the peak positions using the Bragg equation modified for refraction [15] and is found to be $\sim 5.5$ and $\sim 4.7 \mathrm{~nm}$, respectively, for the $\mathrm{CdA}$ and $\mathrm{ZnA}$ multilayers. The alkyl chain tilt angles can be determined considering $2.76 \mathrm{~nm}$ to be the typical length of an arachidate molecule and are found to be $\sim 0$ and $\sim 32^{\circ}$ for the $\mathrm{CdA}$ and $\mathrm{ZnA}$ molecules, respectively.

The GIXD patterns from CdA and ZnA multilayers are shown in Fig. 2(a) and (b), respectively. The GIXD pattern of a CdA multilayer exhibits several peaks in the range $20-50^{\circ}$. All the Bragg peaks could be indexed to (11), (02), (20) and (22) reflections of a centred rectangular 2D lattice and the lattice parameters obtained are: $a=0.47 \mathrm{~nm}$ and $b=0.72 \mathrm{~nm}$. These values are in close agreement with the layer cell dimensions of an ideal close packed herringbone structure, $\mathrm{R}[0,0]$, as proposed by Kitaigorodskii [12]. In contrast, the GIXD pattern of a $\mathrm{ZnA}$ multilayer shows just 
two peaks, a strong peak at $20.1^{\circ}$ and a weak peak at $41.6^{\circ}$. The presence of a single strong peak in the range $20-25^{\circ}$ rules out Kitaigorodskii's close packed structures based on centred rectangular and oblique 2D layer cells, which give rise to two and three strong low order peaks, respectively [16]. The single GIXD peak in the range of $20-25^{\circ}$ shows that the nature of the intralayer molecular packing in the $\mathrm{ZnA}$ multilayer is hexagonal rather than a distorted hexagonal (centred rectangular) as reported earlier [13]. The GIXD peaks can be indexed to (10) and (20) reflections of a hexagonal lattice and the corresponding lattice constant ' $a$ ' is determined to be $0.51 \mathrm{~nm}$. A hexagonal layer cell with $a=0.51 \mathrm{~nm}$ corresponds to a mean molecular area of $0.23 \mathrm{~nm}^{2}$ in the layer plane which is unusually large [3]. This type of molecular packing made up of a hexagonal layer cell in which the molecules are tilted at an angle with respect to the layer normal is distinctly different from the close packed crystal structures of long-chain organic compounds and has not been observed earlier in LB multilayers [3]. It is interesting to note that the contrast in the molecular packing behaviour of $\mathrm{CdA}$ and $\mathrm{ZnA}$ multilayers is observed despite the fact that the mean molecular areas of the $\mathrm{ZnA}$ and $\mathrm{CdA}$ monolayers obtained from the isotherms are nearly equal $\left(\sim 0.20 \mathrm{~nm}^{2}\right)$. It may however be noted that in the case of $\mathrm{ZnA}$ multilayers, the corresponding area per molecule projected in the plane perpendicular to the chains is $0.193 \mathrm{~nm}^{2}$, which is about $5 \%$ larger than the ideal value of $0.184 \mathrm{~nm}^{2}$ corresponding to close packed herringbone structure. Such loose packed structures called 'rotator' phases are known to exist in long chain alkanes at high temperatures [17]. In the rotator phases the molecules are free to rotate about their chain axis, which in the present case is facilitated by the large mean molecular area in the plane perpendicular to the chains. It thus appears that in the $\mathrm{ZnA}$ multilayers, the packing constraints in the headgroup region lead to a hexagonal layer cell with a relatively large mean molecular area. This necessitates tilting of the alkyl chains away from the normal, governed by the interchain molecular forces which do not allow the chain packing density to deviate significantly from the ideal close packed value.
The $2 \mathrm{D}$ crystal structure of the $\mathrm{CdA}$ and $\mathrm{ZnA}$ multilayers has also been studied by molecular resolution AFM. For better statistics several molecular resolution images were obtained from different parts of the sample. Fig. 3 shows a typical $10 \mathrm{~nm} \times 10 \mathrm{~nm}$ image for the CdA multilayer along with the corresponding Fourier transform. The image shows the terminal methyl $\left(\mathrm{CH}_{3}\right)$ group of the topmost layer of $\mathrm{CdA}$ molecules exhibiting the presence of $2 \mathrm{D}$ crystalline order. The 2D Fourier transform of the AFM image exhibits six bright spots and are indexed as (11), $(1 \overline{1}),(\overline{1} 1),(\overline{11}),(02)$ and $(0 \overline{2})$ reflections of a $2 \mathrm{D}$ rectangular lattice. In addition, the $2 \mathrm{D}$ FT also exhibits weak spots corresponding to (01) and $(0 \overline{1})$ reflections. The average values of the lattice constants were found to be, $a=0.49 \pm 0.03 \mathrm{~nm}$ and $b=0.75 \pm 0.04 \mathrm{~nm}$ with an angle of $90 \pm 5^{\circ}$ between them. The presence of weak spots corresponding to $\{01\}$ and $\{10\}$ reflections in the FT image of $\mathrm{CdA}$ multilayers with herringbone structure has been reported earlier by Schwartz et al. [3]. In a $2 \mathrm{D}$ herringbone structure when viewed from top, the molecule in the centre of the rectangle has a different orientation than the molecules at the vertices of the rectangle as shown in Fig. 4. It is clear that the top methyl group is "non-centred' in the rectangle formed by the other four top methyl groups of the corner molecules, which makes the two molecule per unit cell not perfectly centred and results in the appearance of weak spots corresponding to $\{10\}$ and $\{01\}$ reflections. The present case however, represents a slightly different situation as the weak spots corresponding to only $\{01\}$ reflections are observed while the spots corresponding to $\{10\}$ reflections were found to be absent in all the 2D FTs. Such an FT pattern has not been reported earlier and can be understood as follows. Fig. 4(a) and (b) shows schematic diagrams of the two possible types of rectangular (herringbone) lattices viewed from the top, which arise from the two possible orientation of the top methyl group of the central molecule with respect to the four methyl groups of the corner molecules. These two specific orientations of the central molecule can be understood on the basis of glide plane symmetry, in which the glide line is either parallel to the [01]-axis (Fig. 4(a)) or 
the [10] axis (Fig. 4(b)). The absence of $\{10\}$ reflections in the FT image of the CdA multilayer shows that the in-plane $2 \mathrm{D}$ rectangular lattice of the CdA multilayer has a glide line symmetry, with the glide line parallel to the [01] direction. This clearly suggests that the methyl group of the central molecule is not only 'non-centred' but also has a specific orientation with respect to the methyl groups of the corner molecules.

The molecular resolution AFM image of the ZnA multilayer also shows well-defined 2D crystalline lattice. The 2D FT image however shows completely different features. The six nearly symmetric reflections correspond to nearly equal ' $d$ ' values, which confirm the presence of a hexagonal
2D lattice. The value of ' $d$ ' corresponding to six strong reflections averaged over the images taken from various portions of the sample was found to be $0.45 \pm 0.02 \mathrm{~nm}$, which leads to the average value of the $2 \mathrm{D}$ hexagonal lattice constant, ' $a$ ' as $0.52 \pm 0.02 \AA$. These values of ' $d$ ' and ' $a$ ' agree well with the respective values of 0.445 and 0.51 $\mathrm{nm}$ obtained from GIXD measurement of this sample.

\section{Conclusions}

The molecular packing in Cd- and Zn-arachidate divalent fatty acid salt multilayers was stud-

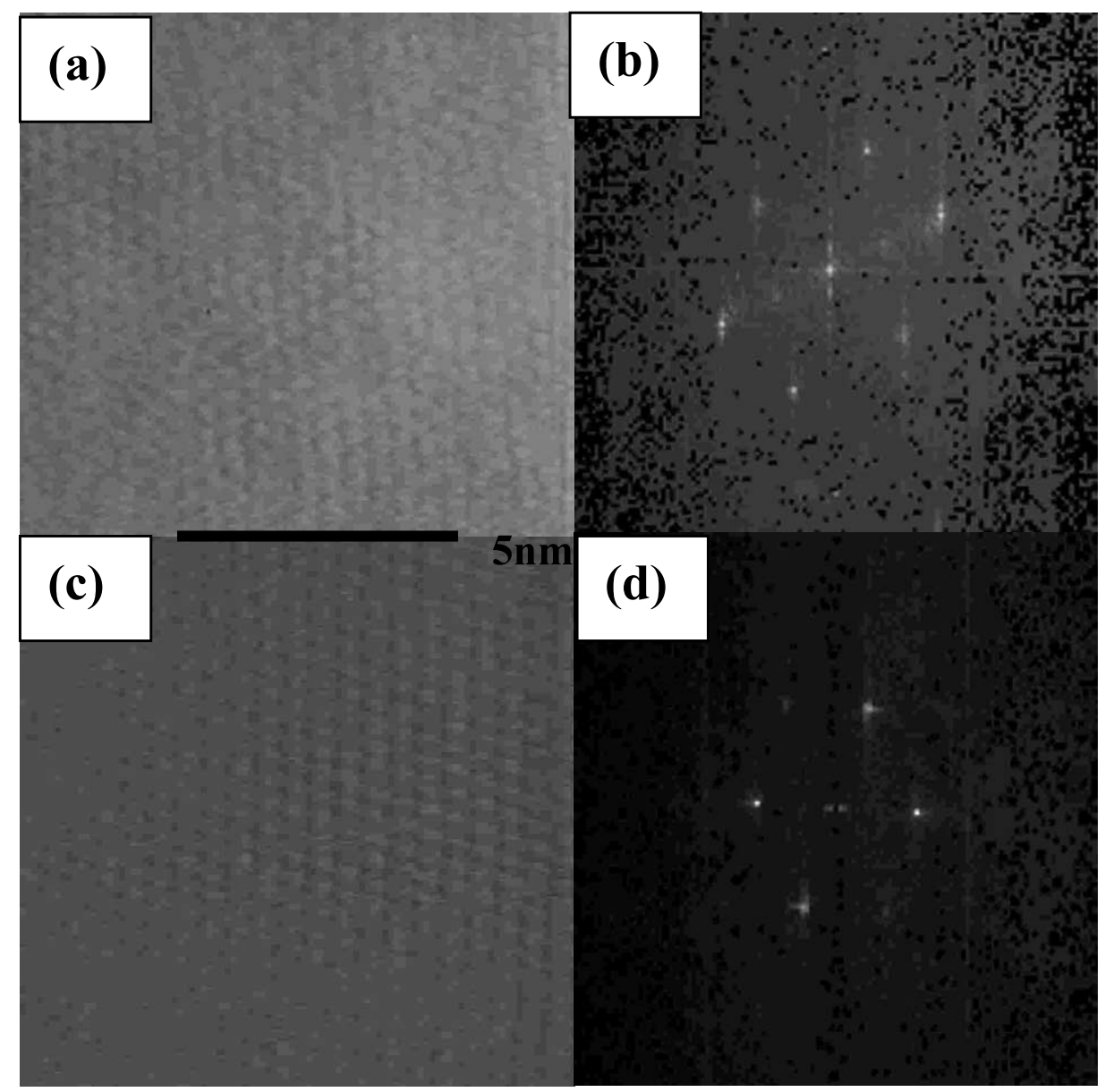

Fig. 3. A $10 \mathrm{~nm} \times 10 \mathrm{~nm}$ molecular resolution AFM image shows the presence of crystalline order in the plane of: (a) CdA; and (c) ZnA multilayers. The Fourier transforms of these images are shown in (b) and (d), respectively. 

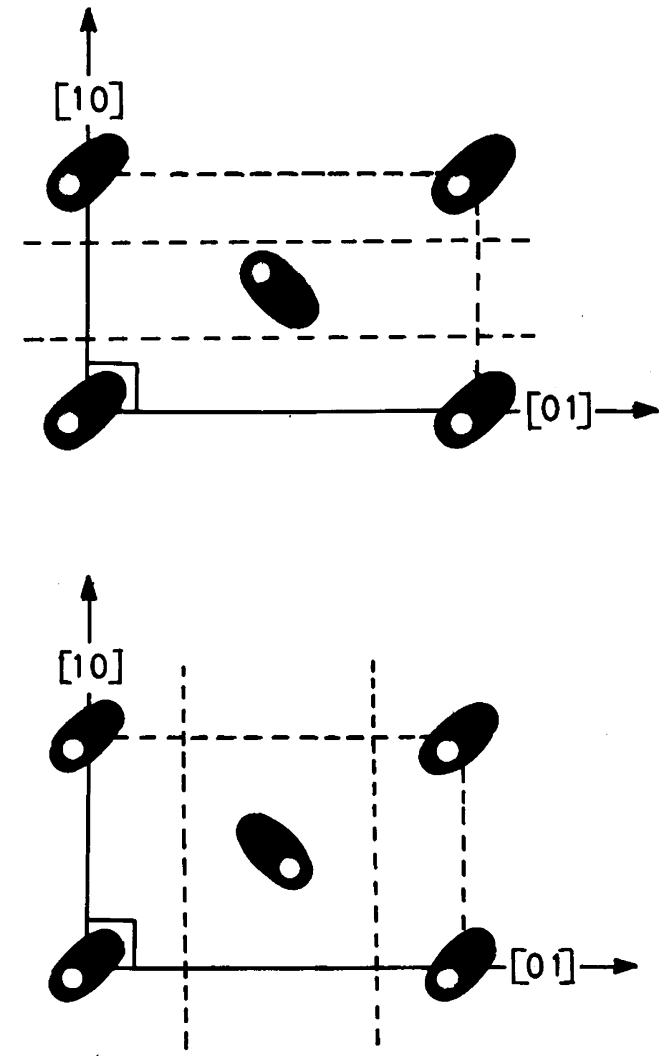

Fig. 4. Schematic of the two possible types of rectangular herringbone structures. The oval represent molecules and the white circles represent terminal methyl groups. The long axis of the oval represents the zigzag plane of the alkane chain viewed from the top. The dashed lines shown parallel to the [10]- and [01]-axis represent glide lines.

ied in detail. In the case of CdA multilayers, the molecules pack in a rectangular herringbone arrangement in the layer plane with the alkyl chains packed nearly perpendicular to the layer plane. This corresponds to $\mathrm{R}[0,0]$, the ideal close packed structure based on orthorhombic subcell packing, as proposed by Kitaigorodskii. The in-plane herringbone packing in $\mathrm{CdA}$ multilayers exhibits a unique feature as observed by AFM. The FT of the images shows clearly two extra, weak spots corresponding to glide plane symmetry suggesting that the methyl group of the central molecule has a specific orientation with respect to the corner molecules in the 2D lattice. The molecular packing in $\mathrm{ZnA}$ multilayers, in contrast, exhibits a loosely packed hexagonal layer cell packing with the alkyl chains tilted at $\sim 32^{\circ}$ to the layer normal. This results in a 'rotator' phase like molecular packing arrangement not reported earlier in LB multilayers.

The above results clearly show that the molecular packing in multilayers of divalent fatty acid salts has a limited dependence on the metal ion electronegativity. The differences in packing behaviour of $\mathrm{ZnA}$ and $\mathrm{CdA}$ multilayers are possibly due to the differences in the nature of metal ion complexation with the acidic headgroup. The results also point towards the complex nature of relationship between the properties of the monolayer on the water surface and the structure of the transferred multilayer.

\section{Acknowledgements}

Financial assistance from the Department of Science and Technology, Government of India and the Indo-Italian Programme of co-operation in Science and Technology is gratefully acknowledged. One of the authors (S.M.) would also like to thank Professor U.C. Sinha, retired professor, Department of Physics, Indian Institute of Technology, Bombay for his valuable suggestions and illuminating discussions on the structure of longchain organic compounds.

\section{References}

[1] G. Roberts (Ed.), Langmuir-Blodgett Films, Plenum Press, New York, 1990.

[2] A. Ulman, An Introduction to Ultrathin Organic Films from Langmuir-Blodgett to Self-Assembly, Academic Press, London, 1991.

[3] D.K. Schwartz, Surf. Sci. Rep. 27 (1997) 241.

[4] S. Garoff, H.W. Deckman, J.H. Dunsmuir, M.S. Alvarez, J.M. Bloch, J. Phys. 47 (1986) 701.

[5] J.E. Riegler, J. Phys. Chem. 93 (1989) 6475.

[6] P. Tippmann-Krayer, R.M. Kenn, H. Möhwald, Thin Solid Films 210/211 (1992) 577.

[7] A. Malik, M.K. Durbin, A.G. Richter, K.G. Huang, P. Dutta, Phys. Rev. B 52 (1995) 11654.

[8] T.A. Barberka, U. Hohne, U. Pietsch, T.H. Metzger, Thin Solid Films 244 (1994) 1061. 
[9] D.G. Wiesler, L.A. Feigin, C.F. Majkrzak, J.F. Ankner, T.S. Berzina, V.I. Troitsky, Thin Solid Films 266 (1995) 69.

[10] J.A. Zasadzinski, R. Viswanathan, I. Madsen, J. Garnaes, D.K. Schwartz, Science 263 (1994) 1726.

[11] D.K. Schwartz, R. Viswanathan, J. Garnaes, J.A. Zasadzinski, J. Am. Chem. Soc. 115 (1993) 7374.

[12] A.I. Kitaigoroskii, Organic Chemical Crystallography, Consultant Bureau, New York, 1961.
[13] R. Viswanathan, L. Madsen, J.A. Zasadzinski, D.K. Schwartz, Science 269 (1995) 51.

[14] A. Dhanabalan, N. Prasanth Kumar, S. Major, S.S. Talwar, Thin Solid Films 327-329 (1998) 787.

[15] B.K. Agarwal, X-ray Spectroscopy, Springer, Berlin, 1979.

[16] J. Als-Nielsen, D. Jacquemain, K. Kjaer, M. Lahav, F. Leveiller, L. Leiserwitz, Phys. Rep. 246 (1994) 251.

[17] E.B. Sirota, D.M. Singer, J. Chem. Phys 101 (1994) 10873. 